\title{
APUNTES SOBRE LAS DIFICULTADES EN LA DETERMINACIÓN DEL ESTATUS CIENTÍFICO DE LA CRIMINOLOGÍA
}

\author{
NOTES ON THE DIFFICULTIES IN DETERMINING THE SCIENTIFIC STATUS OF \\ CRIMINOLOGY
}

Sebastián Alonso Agüero San Juan*

Fecha de recepción: 13 de mayo de 2010.

Fecha de aceptación: 10 de junio 2010.

\begin{abstract}
Resumen
El presente artículo conf囚gura una panorámica de los problemas que tiene enjuiciar a la criminología como ciencia. Se revisan los principales criterios usados normalmente para asignar el rótulo de 'ciencia' a una determinada disciplina y se concluye con una propuesta de ordenación de tales criterios.
\end{abstract}

Palabras clave: criminología, pseudociencia, disciplinas científic
Abstract
This article sets an overview of the problems with prosecuting criminology as a science. We review the criteria normally used to assign the label of 'sci- ence' to a particular discipline and concludes with a proposal for manage- ment of these criteria.

Key words: criminology, pseudoscience, scienti \c disciplines .

Máster en Ciencias Jurídicas, Universidad Pompeu Fabra, aguero.sj@gmail.com 
"...Algo así como un parásito, la criminología se une a sus materias anfitrionas (en particular, derecho, psicología, psiquiatría y sociología) y de ellas extrae métodos, teorías y credibilidad...".

Stanley Cohen 1988.

"El crimen a veces se caracteriza como una ciencia social de atracción turística, un Taj Mahal o Torre de Pisa, que visita todo el mundo una sola vez. Por lo tanto los teóricos más importantes, como Durkheim, Parsons o Merton han hecho apariciones claves, pero breves en la escena del crimen".

Frances Heidnsohn 1989.

\section{Introducción}

El estudio de manuales dedicado a una disciplina en particular entrega una visión, no solo de conocimientos básicos sobre la materia que permiten, una vez aprendidos, introducirse con mayor profundidad en el área, sino, también, de cuáles son las principales interrogantes que atormentan a dicho ámbito del conocimiento.

En este último sentido, al revisar libros de textos sobre Criminología es posible constatar, principalmente, en sus primeros capítulos, una excesiva cantidad de argumentos destinados a justificar su carácter o estatus científico ${ }^{1}$, mientras que, también, se observa, la exposición de una relación problemática entre 'Ciencia' y 'Criminología'2. Cabe señalar que esto sucede sin perjuicio del tratamiento realizado por algunos autores que, sin introducirse en la discusión, dan por sentado el carácter científico de la Criminología ${ }^{3}$.

Los criminólogos imbuidos en la problemática, por lo general, presentan una serie de argumentos destinados a obtener el reconocimiento científico de la 'Crimino-

1 En sentido: García-Pablos de Molina, A. Criminología: Una introducción a sus fundamentos teóricos (Quinta edición ed.). Valencia: Tirant lo Blanch, 2005; Garrido, V., Stangeland, P. \& Redondo, S. Principios de Criminología. Valencia: Tirant lo Blanch, 2006; Lanier, M. M. \& Stuart, H. Essential Criminology (Tercera edición ed.). Philadelphia: Westview Press, 2010; Serrano Maíllo, A. Introducción a la Criminología (Quinta edición ed.). Madrid: Dykinson, 2008.

2 Siguiendo esta línea: Coleman, C. \& Norris, C. Introducing Criminology. Portland: Willan Publishing, 2000; Kaiser, G. Introducción a la Criminología (Séptima edición ed.). (N. J. Rodríguez \& J. M. Rodríguez Devesa, Trads.) Madrid: Dykinson, 1988; Newburn, T. Criminology. Portland: Willan Publishing, 2007; \& Tierney, J. Criminology: theory and context (Segunda edición ed.). London: Pearson Education Limited, 2006.

3 Con este enfoque: Cid Moliné, J. \& Larrauri Pijoan, E. Teorías Criminológicas. Barcelona: Bosch, 2001; y Roldán Barbero, H. Introducción a la investigación criminológica. Granada: Editorial COMARES, 2009. 
logía'. En concreto, su estructura argumental puede ser caracterizada por tres elementos: (1) su objeto de estudio lo constituye la realidad empírica, (2) su metodología es la utilizada de manera general en las ciencias sociales, y (3) su producto es un conocimiento válido, fiable y contrastable. Sin embargo, existe otro grupo de autores, más cuidadosos al momento de otorgar carácter científico a la Criminología, para quienes resulta esencial señalar la diversidad de enfoques disciplinarios utilizados en Criminología, pues ello conlleva ciertas indeterminaciones internas que dificultan aún más la obtención del atributo científico.

No obstante, tomando algo de distancia de estas formas de abordar el estatus científico de la Criminología, en el presente trabajo se pretende exponer y desarrollar las principales dificultades que deben sortear los criminólogos al momento de atribuir o designar con la palabra 'ciencia' a su objeto de estudio.

Por consiguiente, la presentación de los inconvenientes se efectuará realizando un tratamiento separado de las nociones: 'Criminología' y 'Ciencia', donde se explicita la indeterminación conceptual de ambas, y luego se destacan algunos elementos considerados como integrantes de su contenido significativo, así, en base a ellos, realizar una contrastación entre ambas nociones que permita proponer un respuesta a la cuestión de si la Criminología posee (o no) estatus científico.

\section{Sobre la Criminología}

La palabra 'Criminología' presenta algunos problemas relativos a su denotación, es decir, a los objetos a los cuales se refiere. Esta indeterminación se manifiesta o es consecuencia de que la disciplina no ha circunscrito definitivamente su objeto de estudio. En concreto, los manuales estudiados difieren en cuanto a lo que debe entenderse por Criminología. Consciente de estas diferencias Tierney sostiene que "...la complejidad de los contornos de esta investigación aparentemente simple, puede destacarse, señalando que no es inusual que criminólogos de avanzada edad aún debatan sobre estas cuestiones ${ }^{4}$. Aun así, es posible agrupar a los autores en atención a los aspectos que consideran como esenciales dentro del estudio criminológico.

Un primer grupo propone que la Criminología tiene por objeto de estudio: el delito o crimen, el delincuente o infractor, la víctima, y el proceso de criminalización o control social ${ }^{5}$. Un segundo grupo centra sus investigaciones en: el crimen, el

4 Tierney, Criminology: theory and context, op. cit, pág., 11.

5 En este sentido: Cid y Larrauri, Teorías Criminológicas, op. cit.; García-Pablos, Criminología: Una introducción a sus fundamentos teóricos, op. cit.; y Roldán, Introducción a la investigación criminológica, op. cit. 
delincuente y el sistema penal o de control del delito ${ }^{6}$. Un tercer grupo sostiene que el saber criminológico se sustenta en dos pilares: el comportamiento delictivo y la reacción social a dichos comportamiento, para unos ${ }^{7}$, y el delito y el delincuente como fenómenos globales, para otros ${ }^{8}$. Finalmente, un cuarto grupo se centra en el delito o crimen, entendido como un fenómeno omnicomprensivo de casi todas las otras materias recién enunciadas ${ }^{9}$.

La agrupación de autores, por concepciones criminológicas, permite proponer o atribuir un campo de estudio difuminado a la Criminología, el cual de manera centrífuga se desvanecería desde el estudio del crimen o delito (situado en el centro), por convertirse en un lugar común a todas las propuestas, hacia las investigaciones sobre víctimas (ubicadas en la periferia), por ser elementos contingentes, y atravesando al delincuente y el control social (posiciones en la zona media), por obtener un consenso relativo.

Ahora bien, la propuesta anterior no debe pasar por alto otra indeterminación, presente en los planteamientos criminológicos, derivada de los significados atribuidos a los términos utilizados para delimitar su objeto. En este sentido, las afirmaciones de Coleman y Norris sobre 'crimen' y 'criminal' resultan sumamente ilustrativas: “... los criminólogos no están necesariamente de acuerdo sobre la definición de esos dos términos básicos, en lugar de darlos por sentados, algunos criminólogos insisten en que dichos términos deben ser vistos como problemáticos. Cuando los términos crimen y criminal son usados, nosotros debemos ser conscientes de la existencia de usos alternativos de esos términos clave. Los términos crimen y criminal no deben ser aceptados en sentido literal, y la consideración de la definición de ellos es una parte clave del tema de la disciplina..."10. De la misma manera, todos los otros términos utilizados para establecer el campo de los estudios criminológicos (víctima, control social, sistema penal) adolecen de este tipo de indeterminación, por tanto, se dificulta aún más la labor de delimitación conceptual.

Una vez enunciados los objetos de estudio de la disciplina, algunos autores hacen referencia a dos características distintivas de la Criminología, a saber: su carácter empírico y la diversidad de enfoques disciplinarios utilizados. Ambas características se originan en sus primeros años. La primera, habría entregado autonomía y cierto 'estatus científico' a los estudios criminológicos de mediados del siglo XIX, debido,

\footnotetext{
Siguiendo esta línea: Kaiser, Introducción a la Criminología, op. cit.; y Newburn, Criminology, op. cit.

Contestes con esta propuesta: Garrido, Stangeland y Redondo, Principios de Criminología, op. cit.

8 Este planteamiento es formulado por: Coleman \& Norris, Introducing Criminology, op. cit.; y op. cit.

9 Este camino es recorrido por: Lanier \& Stuart, Essential Criminology, op. cit. \& Tierney, Criminology: Theory and context, op. cit.

10 Coleman \& Norris, Introducing Criminology, op. cit., pág. 13.
} 
principalmente, a la generalización y valoración realizada por el positivismo filosófico del método empírico, el cual vino a desplazar al racionalismo abstracto, formal y deductivo del mundo clásico ${ }^{11}$. Por su parte, la segunda comienza a configurarse con el descubrimiento realizado por la escuela positivista-biológica de la 'persona delincuente', pues dicho hallazgo facilitó sustantivamente la introducción de diversos profesionales a este emergente campo de estudio ${ }^{12}$.

El carácter experiencial comprende la utilización de métodos empíricos (principalmente observación y experimentación), aplicables a los objetos de estudio, para así generar conocimientos fiables y seguros, siempre que dichos conocimientos (teorías o hipótesis) puedan ser verificados o falseados ${ }^{13}$. Este rasgo vincula directamente a la Criminología con las ciencias empíricas, en el sentido de obtener su objeto de estudio del mundo real, verificable y mensurable, en otras palabras, se apropia de una parte de la realidad susceptible de explicación ${ }^{14}$. En este mismo orden de ideas se inserta el tratamiento realizado, por algunos de los textos estudiados, de los planteamientos relativos a los diversos métodos y técnicas de investigación, utilizados por la Criminología, para estudiar la realidad social. Las cuales son agrupadas en dos grandes bloques: (1) cuantitativos, destinados a explicar las causas, génesis o desarrollo de un fenómeno (estudios estadísticos -policiales, judiciales y penitenciaras-, encuestas de victimización, estudios de autodenuncia, entre otros); y (2) cualitativos, encaminados a comprender las profundas claves de un fenómenos (entrevista en profundidad, los grupos de discusión, la observación participante, etc. $)^{15}$.

La diversidad de enfoques disciplinarios utilizados en el saber criminológico, proporcionan una visión de la Criminología como confluencia de diversas formas de construir conocimiento o afluentes. Los principales saberes confluyentes son: la antropología, biología, economía, geografía, historia, filosofía, derecho, ciencias políticas, psiquiatría, psicología y sociología ${ }^{16}$. El hecho de confluir diversas formas de reconstruir la realidad produce, fundamentalmente, dos complicaciones: el cómo abordar esta variedad disciplinar y el cómo mitigar las contiendas disciplinares.

La primera complicación se manifiesta en el enfrentamiento entre la opción multidisciplinaria frente a la interdisciplinar. Lo multidisciplinar hace referencia a un tipo

\footnotetext{
11 Cid y Larrauri, Teorías Criminológicas, op. cit., pág. 12; y García-Pablos, Criminología: Una introducción a sus fundamentos teóricos, op. cit.; pág. 38.

12 Op. cit., pág. 42.

13 García-Pablos, Criminología: Una introducción a sus fundamentos teóricos, op. cit.; pág. 39.

14 Op. cit., pág. 40.

15 Cid y Larrauri, Teorías Criminológicas, op. cit., págs. 20-21; y García-Pablos, Criminología: Una introducción a sus fundamentos teóricos, op. cit.; págs. 44-48.

16 Coleman \& Norris, Introducing Criminology, op. cit., pág. 15.
} 
de investigación que abarca o afecta a varias disciplinas, posibilitando que cada una de estas contribuya con sus propios supuestos sobre la naturaleza humana y social, es decir, los objetos de estudio pueden ser visualizados desde diferentes perspectivas $^{17}$. Por otro lado, lo interdisciplinario sugiere una actividad que se realiza con la cooperación de varias disciplinas, es decir, integrando los conocimientos en un todo distinto $^{18}$, para así concentrar las cuestiones relevantes de las otras disciplinas en los problemas criminológicos y condicionar a los investigadores a mantenerse lo suficientemente abierto para contemplar otras especialidades. Frente a ambas propuestas la doctrina toma caminos diversos. Por un lado, se sostiene que la Criminología no sería interdisciplinaria, ya que pocos autores han intentado dicha integración, siendo más apropiado el calificativo de multidisciplinar ${ }^{19}$, y por otro, se reconoce la necesidad de una investigación multidisciplinar, pero se considerada como alternativa real la interdisciplinariedad, ya que pese a exigir del investigador una mayor integración de conocimientos, debiera ser considerada como el destino de la Criminología empírica ${ }^{20}$.

La segunda complicación, derivada de diversos afluentes epistémicos, produce una serie de conflictos y rivalidades internas, porque una de ellas obtenga el predominio sobre otras. Coleman y Norris sugieren que "... cada una de estas disciplinas tiende a: (1) tener sus propios supuestos, ideas y conceptos básicos, (2) un banco de conocimientos compartidos, (3) un enfoque particular de la investigación, (4) favorece métodos de investigación, y (5) una visión del mundo que lleva a tipos particulares de implicaciones políticas" 21 . Si las afirmaciones de los autores ingleses son correctas, resulta evidente la generación de conflictos entre las disciplinas, que pueden ser formulados, siguiendo a Tierney, como competencia sobre: el foco de los estudios, la agenda a seguir, las teorías a emplear, los métodos de investigación y la orientación política ${ }^{22}$.

En definitiva el estado actual de la Criminología impide presentar de manera clara y precisa: cuál es su objeto (u objetos) de estudio, cuál es el contenido atribuido a dicho objeto (u objetos), cuál es la manera de abordar la confluencia de otras disciplinas y cuál es la forma de atenuar las rivalidades entre ellas. Por tanto, con estas indeterminaciones no solo deben lidiar los criminólogos, para quienes proporcionar contornos conceptuales más nítidos puede constituir un deber, sino, también, para

Lanier \& Stuart, Essential Criminology, op. cit., pág. 18; \& Newburn, Criminology, op. cit., pág. 5.

18 Garrido, Stangeland y Redondo, Principios de Criminología, op. cit., pág. 47; y Kaiser, Introducción a la Criminología, op. cit., pág. 32.

19 Lanier \& Stuart, Essential Criminology, op. cit., pág. 18.

20 Kaiser, Introducción a la Criminología, op. cit., pág. 32.

21 Coleman \& Norris, Introducing Criminology, op. cit., pág. 15. La numeración no se encuentra en el texto original.

22 Tierney, Criminology: Theory and context, op. cit., págs. 11-12. 
quienes desean constatar la existencia de un estatus científico por parte de la Criminología.

\section{Ciencia}

La palabra Ciencia presenta serias dificultades en el proceso de delimitar su alcance. Así, en el uso común presenta problemas de ambigüedad (proceso-producto) y vaguedad (denotación notoriamente imprecisa) que repercuten directamente en el ámbito académico, donde la actividad de entregar condiciones suficientes y necesarias plausible para la ciencia, constituye una tarea sumamente ardua, al punto de que muchos filósofos de la ciencia hayan decidido abandonar el problema de la demarcación, es decir, el problema de proponer tales condiciones ${ }^{23}$.

A causa de ello, se intentan abordar dichas dificultades proponiendo asumir 'Ciencia' como un concepto racimo (cluster concept), el cual permite la existencia de variadas actividades relacionadas entre sí por semejanza o analogía y que, pese a actuar bajo su nombre, ninguna por sí sola es la 'ciencia' como tal ${ }^{24}$. Esta propuesta solo le proporciona una forma o estructura al concepto resultando necesario proporcionarle un contenido. Para satisfacer esta exigencia, se comienza contrastando 'Ciencia' con otro tipo de conocimientos (sentido común y seudociencia), para así obtener ciertos contornos científicos que permiten diferenciarla de otras clases de información. Luego, en atención a ciertos rasgos del saber criminológico, en un primer momento, se expone las características esenciales y principales de las ciencias empíricas, para finalmente, presentar dos marcos generales de referencia (empirista y humanista), que han orientado históricamente la investigación social desde su nacimiento.

Todo este procedimiento, destinado a llenar de contenido el concepto 'Ciencia', proporciona un panorama general de las dificultades a las cuales se deben ver enfrentados los filósofos de la ciencia al momento de determinar, establecer o proponer las condiciones suficientes y necesarias plausibles para la 'Ciencia'.

1) Ciencia y sentido común: Ernest Nagel, en su libro La estructura de la ciencia (1989), sostiene la existencia de un núcleo o significado seguro de la palabra Ciencia, percibido al destacar las diferencias importantes y reconocibles existentes en el uso de la palabra. Estas son resaltadas al comparar sentido común y Ciencia (como tipos de información sobre las cosas), señalando como elementos del significado seguro: a) pretender hallar explicaciones a los hechos, estableciendo cierta relación de depen-

\footnotetext{
23 Plantinga, A. (20 de febrero de 2007), Religion and Science. Recuperado el 1 de abril de 2010, de Stanford Encyclopedia of Philosophy: http://plato.stanford.edu/entries/religion-science/

24 Op. cit.
} 
dencia entre proposiciones aparentemente desvinculadas y poniendo de manifiesto sistemáticamente las conexiones existentes entre temas de información variados; b) estar movilizada por juicios antagónicos, en la medida que al introducir explicaciones sistemáticas de los hechos, discernir entre condiciones y consecuencias de los sucesos, y manifestar las relaciones lógicas entre las proposiciones, (la ciencia) puede atacar las bases de dichos juicios e incluso unificar propuestas; c) intentar disminuir las indeterminación del lenguaje corriente, sometiéndolo a modificaciones, para que sus enunciados sean susceptible de pruebas o críticas, e incorporarlos en sistemas explicativos lógicamente integrados; d) intentar no considerar la valoración humana y los valores inmediatos de las cosas en la determinación de las relaciones de dependencia entre las cosas; e) proponer conceptos abstractos para lograr una explicación general de cosas cualitativamente diversas, expresando las propiedades estructurales del concepto sin hacer referencia a sus cualidades y relaciones individuales, pudiendo así elaborar sistemas explicativos para grandes conjuntos de fenómenos diversos; y f) exponer sus afirmaciones cognoscitivas, repetidamente, a desafíos en condiciones cuidadosamente controladas para obtener datos observacionales críticamente probatorios. Por último, el autor reconoce que las características recién enunciadas se justifican en la medida que adhieren a una política definida de cómo obtener y evaluar elementos de juicio, agregando, que el registro histórico y los logros obtenidos han garantizado la superioridad de esta política ${ }^{25}$.

2) Ciencia y seudociencia: Sven Ove Hansson, en su artículo sobre Sciencie and Pseudo-Science, enuncia y cuestiona los criterios de demarcación científica desarrollados, considerándolos como parte integrante de la tarea de determinar ¿qué creencias son epistémicamente justificadas? Por tanto, postula que es posible encontrar criterios de demarcación en: (1) los positivistas lógicos, quienes sugieren que una declaración científica se distingue de una metafísica por ser, al menos, susceptible de verificación ${ }^{26}$; (2) el falsacionismo, la falsabilidad (criterio sugerido por Karl Popper) es condición necesaria y suficiente, pues una oración (o teoría) es empírico-científica si y solo si es falseable ${ }^{27}$; (3) la resolución de puzles, el criterio de demarcación propuesto por Thomas Kuhn consiste en la capacidad de la 'Ciencia' para resolver puzles o enigmas realizados durante el desarrollo científico normal; (4) el criterio basado en el progreso científico, en esta categoría destaca George Reisch, quién exige a las disciplinas científicas que se integren adecuadamente en o con otras ciencias, pues varias disciplinas científicas poseen fuertes interconexiones basadas en metodolo-

25 Nagel, E. (1989), "La ciencia y el sentido común". En E. Nagel, La estructura de la ciencia (N. Miguez, Trad., págs. 15-26). Barcelona: Paidós.

26 Este criterio es vinculado con la idea que el sentido de una proposición es su método de verificación. Verificación entendida como el proceso de añadir hechos observacionales para corroborar hipótesis, pudiendo quedar estas consolidadas.

27 Una sentencia (teórica) es falesable si y solo si es lógicamente contradictoria con otra sentencia (empírica) que describe un evento lógicamente posible, que puede ser lógicamente posible observar. 
gía, teorías, etc.; y (5) las normas epistémicas, de Robert K. Merton, señalan que la ciencia tiene un ethos, expresable en cuatro imperativos institucionales: universalismo (exigencia de comprobación), comunismo (conocimiento público), desinterés (neutralidad motivacional), y el deber de plasmar los otros imperativos; para concluir señalando que en la comunidad académica habría acuerdos generalizados solo sobre cuestiones puntuales, existiendo aún desacuerdo sobre las generalizaciones ${ }^{28}$.

3) Ciencia material o empírica: Mario Bunge, intentando caracterizar el conocimiento y la investigación científica material o empírica, propone como rasgos esenciales del conocimiento obtenido por esta la racionalidad y objetividad. De manera que el conocimiento es racional si (1) está constituido por conceptos, juicios y raciocinios, es decir, ideas capaces de visualizar modelos y hacer operaciones; (2) esas ideas pueden cambiarse por un conjunto de reglas lógicas, para producir nuevas ideas (inferencia deductiva); y (3) pueden organizarse en sistemas, esto es, en conjuntos ordenados de proposiciones (teorías). Por su parte, el conocimiento de la realidad es objetivo si (1) concuerda aproximadamente con su objeto, es decir, busca alcanzar la verdad fáctica; y (2) verifica la adaptación de las ideas a los hechos, a través de una particular interacción con estos últimos (observación y experimento), que es controlable y hasta cierto punto reproducible ${ }^{29}$.

Luego, Bunge propone catorce características principales de la ciencia material, derivadas de los rasgos esenciales, las cuales pueden agruparse en tres grupo: (1) las relativas al conocimiento científico, el cual es: fáctico, al intentar describir los hechos tal como son con independencia de su valor; trasciende los hechos, en la medida que los descarta, produce y explica; claro y preciso en la forma en la cual se expresa; comunicable, esto es, expresable y público; verificable, pues la comprobación de sus hipótesis involucra la experiencia; sistemático, al ser un sistema de ideas conectadas lógicamente entre sí; general, al ubicar hechos singulares en pautas generales y enunciados particulares en esquemas amplios; legal, ya que inserta hechos singulares en pautas generales Ilamadas leyes; y predictivo, al transcender el presente intentado reconstruir el pasado e imaginar el presente. (2) Las relativas a la ciencia, que se caracterizaría por ser: analítica, al intentar descubrir los elementos que componen cada totalidad, y las interconexiones que explican su integración; explicativa, pues explica los hechos en términos de leyes, y las leyes en términos de principios; abierta, al no reconocer barreras que limiten a priori el conocimiento; y útil, como herramienta para domar la naturaleza y remodelar la sociedad. (3) Y las relativas a la investigación científica, que la presentan como: especializada, debido al enfoque analítico con el cual se abordan los problemas; y metódica, ya que las investigaciones se fundan so-

28 Ove Hansson, S. (3 de septiembre de 2008), Sciencie and Pseudo-Science. Recuperado el 20 de marzo de 2010, de Stanford Encyclopedia of Philosophy: http://plato.stanford.edu/entries/pseudo-science/

29 Bunge, M. (1985), “¿Qué es la ciencia?”. En M. Bunge, La ciencia su método y filosofía (págs. 8-36). Buenos Aires: Ediciones Siglo Veinte. 
bre el conocimiento anterior y proceden conforme reglas y técnicas que han resultado eficaces en el pasado pero que son perfeccionadas continuamente ${ }^{30}$.

4) Ciencia social: Piergiogio Corbetta, en su libro Metodología y técnicas de investigación social, caracteriza las ciencias sociales como condicionadas por dos grandes marcos de referencia: el positivismo y el humanismo, que establecieron los paradigmas ${ }^{31}$ fundacionales de la investigación social, proporcionando procedimientos operativos en causadores del desarrollo de las disciplinas empírico-sociales. El autor dilucida dichos marcos y su constatación en las ciencia sociales, formulando un esquema comparativo basado en: ¿existe la realidad social? (ontología), ¿es conocible? (epistemología) y ¿cómo puede ser conocida? (metodología), presentando sus respuestas dentro tres categorías: Positivismo, Neo y Postpositivismo, e Interpretacionismo. El estudio de estos marcos permite entender cómo las ciencias sociales, en general, responden a dichas preguntas ${ }^{32}$ : Ontología, existe una realidad social externa al sujeto susceptible de ser conocida, de manera: a) objetiva en su esencia real, b) imperfecta por las imperfecciones del conocimiento humano, o c) variada, en tanto múltiples y diversas son las perspectivas que las personas utilizan para interpretar los hechos sociales; Epistemología, es posible conocer la realidad considerando: a) una independencia entre sujeto y objeto (dualismo), la no influencia recíproca (objetividad), y descubrir leyes deterministas (causa-efecto), b) elementos de perturbación introducidos por el investigador y sus efectos, la objetividad como ideal regulativo, la falsabilidad como criterio de validación empírica de las afirmaciones, y leyes generalizadoras, probabilísticas y temporales, c) como objetivo la comprensión del comportamiento del individuo y utilizando abstracciones y generalizaciones (tipos ideales y enunciados de posibilidad); Metodología, para conocer la realidad social se pueden emplear: a) métodos y técnicas de investigación inductivas, con pretensiones matemática y basadas en la experimentación, b) instrumentos con soportes positivos (operacionalización, cuantificación y generalización), pero considerando técnicas cualitativas; c) actitudes de interacción entre sujeto y objeto (consideradas como la base del progreso cognoscitivo), para poder comprender el significado de las acciones, por medio de técnicas de investigación cualitativas y subjetivas ${ }^{33}$.

En resumen, los intentos de determinación del contenido científico, diferenciándolo de otros tipos de conocimientos, y luego, una vez delimitados sus contornos

30 Op. cit.

31 Paradigma es entendido como una perspectiva teórica fundada sobre adquisiciones precedentes a la disciplina misma, que actúa dirigiendo la investigación, desde la identificación y elección de los hechos relevantes a estudiar, hasta la formulación de las hipótesis depositarias de la explicación del fenómeno observado, y preparar necesarias técnicas de investigación empíricas.

32 La letra a) corresponde a la posición positivista, la letra b) a la postura neo y postpositivistas; y la letra c) representa los planteamientos de los interpretacionistas.

33 Corbetta, P. (2003), Metodología y técnicas de investigación social (M. Díaz Ugarte y S. Díaz Ugarte, Trads.). Madrid: McGraw-Hill. 
diferenciadores, desarrollando los rasgos fundamentales de un área del conocimiento científico, como es el empírico-social, pone de manifiesto la variedad de incertidumbres existentes en torno a qué clase o tipos de conocimientos pueden subsumirse dentro de la categoría científica.

\section{Ciencia y Criminología}

La cantidad y diversas indeterminaciones existentes tanto en el terreno criminológico como en el científico, constituyen por sí mismas una seria dificultad en la tarea de determinar o establecer el estatus científico de la 'Criminología', la cual no debe ser olvidada ni obviada por quienes emprenden dicha actividad.

Pero sin perjuicio de lo anterior, es posible utilizar los acercamientos y delineamientos del concepto 'Ciencia', considerándolos como filtros útiles en la contrastación de los conocimientos con pretensiones de cientificidad. En este sentido, estos pueden agruparse en etapas, considerando su grado de especificidad, lo cual permitiría establecer si un conocimiento es ciencia (en sentido general), si es empírica (como una clase de ciencia) y si es social (como especie de empírica). De esta forma, es posible contrastar las nociones de 'Criminología" a las nociones de 'Ciencia' para apreciar si las primeras participan o contienen características de las segundas.

En este orden de cosas, los criterios de la primera etapa pueden sintetizarse en las siguientes afirmaciones sobre el conocimiento científico: (1) pretende explicar los hechos, fenómenos o realidades, estableciendo conexiones entre proposiciones, materias o hechos aparentemente desvinculados y presentándolas de manera sistemática; (2) intenta disminuir la imprecisión del lenguaje corriente al establecer sus propuestas; (3) es valorativamente neutro, pretendiendo no considerar valoraciones humanas o de las cosas; (4) propone conceptos abstractos, capaces de explicar de manera general cosas cualitativamente diversas; (5) necesita de la comprobación de sus proposiciones, la cual se puede realizar por verificación o falseación; y (6) puede integrarse en otros sistemas explicativos lógicamente integrados o áreas del conocimiento reconocidas como científicas.

La segunda etapa está compuesta por afirmaciones distintivas de las ciencias empíricas: (1) sus explicaciones de los hechos pretenden concordar con ellos (verdad fáctica); (2) las propuestas son contrastadas con los hechos (observación y experimentación); (3) establece leyes, ya que inserta hechos singulares en pautas generales Ilamadas leyes (hechos-leyes-principios); (4) son predictivas, al transcender el presente intentado reconstruir el pasado e imaginar el futuro; (5) intentan descubrir y explicar los elementos e interconexiones que componen una totalidad (analíticas); (6) persiguen la utilidad, siendo herramienta para domar la naturaleza y remodelar la sociedad; y (7) serían metódicas, al planificar sus pasos a seguir. 
La tercera y última etapa sugiere los rasgos esenciales de una ciencia social: (1) reconoce la existencia de una (o varias) realidad social externa al sujeto; (2) considera posible conocer dicha realidad de manera independiente y objetiva o de modo comprensivo, pudiendo establecer para ello leyes (deterministas o probalístico-temporales) o generalizaciones y abstracciones; y (3) para conocer la realidad social emplean métodos y técnicas de investigación cuantitativas o cualitativas.

Estos criterios de comprobación son enfrentados por las nociones criminológicas, que sugieren una ubicación dentro de las ciencias empírico-sociales; en consecuencia, el discurso criminológico deberá ser contrastado en las dos categorías de científico y empírico-social.

La primera categoría es superada en base a que la Criminología: (1) al pretender explicar sus objetos de estudios (delito, delincuente, víctima y procesos de criminalización) da a conocer causas o motivos de hechos, estados o realidades; (2) en el afán de determinar con exactitud su campo de estudio, problematiza sus objetos con la finalidad de obtener una mayor pulcritud conceptual; (3) posee una variada gama de conceptos abstractos para explicar cosas distintas y particulares, como son: delito, delincuencia, reacción social, política criminal, entre otros; (4) somete a comprobación, de manera minuciosa y reiterativa, las afirmaciones surgidas en su seno, por ejemplo: las teorías criminológicas propuestas a lo largo de su historia, durante muchos años han sido objeto de investigaciones destinadas a verificarlas o falsearlas ${ }^{34}$; $(5)$ por su carácter multi o interdisciplinar los conocimientos emanados de esta área pueden fácilmente intercalarse en otras disciplinas con las cuales comparten algunos supuestos básicos; y (6) pese a ser discutida su neutralidad, en el sentido de estar condicionado su estudio por grupos de poder que establecen las normas dentro de una sociedad ${ }^{35}$, estos ejercicios del poder, mediante proceso de etiquetamiento de actos y personas como criminales (criminalización), tienden a reflejar diferentes poderes e intereses particulares dentro de una sociedad, lo cual más que manipular amplía los horizontes de investigación ${ }^{36}$.

Por su parte, la segunda categoría es satisfecha por el conocimiento criminológico que: (1) es esencialmente fáctico, como se ha sostenido y desarrollado en el apartado II; (2) propone explicar los hechos objeto de estudio (fenómeno delictivo) formulando propuestas explicativas de su acaecimiento (teorías criminológicas), para intentar comprender su surgimiento o prevenir su ocurrencia, para luego ser sometidas a comprobación detallada; (3) pretende prestar utilidad, principalmente en la prevención de los delitos y en el sistema penal ${ }^{37}$; (4) emplea en sus investigaciones

\footnotetext{
Cid y Larrauri, Teorías Criminológicas, op. cit., pág. 254.

Coleman \& Norris, Introducing Criminology, op. cit., pág 7.

6 Newburn, Criminology, op. cit., pág. 8.

37 Cid y Larrauri, Teorías Criminológicas, op. cit., págs. 2-23.
} 
métodos cuantitativos y cualitativos (como se señala en el apartado II), para plasmar de la manera más acabada sus objetos de estudio; (5) solo cobra sentido en la medida que reconoce la existencia de una realidad externa susceptible de ser conocida; y (6) posee un desarrollo histórico estrechamente vinculado y similar al experimentado por los marcos de referencia de las ciencias sociales, pues adquiere autonomía bajo el amparo de la filosofía positivista, adhiriendo a sus postulados (positivismo biológico), luego, manteniéndose dentro del mismo marco, se matizan algunos discursos para dar repuestas más precisas a los objetos de estudio (escuelas sociológicas) y, finalmente, se produce un cambio de orientación respecto de lo anterior, dominado por el paradigma causal (teoría del etiquetamiento).

Para finalizar y entregar una mayor profundidad, es posible agregar a la contrastación anterior, los argumentos sostenidos por los criminólogos (en sus manuales o libros de textos) para justificar el estatus científico de la disciplina que desarrollan. Estos fundamentos aluden a que la Criminología: (1) posee un objeto de conocimiento, un métodos de estudio y un sólido cuerpo doctrinal sobre el fenómeno delictivo, compuesto por información válida, fiable y contrastable ${ }^{38}$; (2) sus seguidores adhieren estrictamente al método científico, construyendo así un conocimiento criminológico desde interrelaciones lógicas, bases teóricas y testeando empíricamente las hipótesis que son materia de nuevos análisis, mediante métodos cuantitativos o cualitativos ${ }^{39}$; y (3) utiliza métodos de investigación prestados de otras ciencias sociales y desarrolla los propios, disponiendo de un gran conjunto de conocimientos sobre la delincuencia y sobre su control, adquiridos de múltiples investigaciones empíricas y del tema de estudio preocupa a cualquier sociedad, generando gran interés público ${ }^{40}$.

En base a todo lo anteriormente señalado, con salvedades y recaudos, es posible dar una respuesta afirmativa a la pregunta ¿es la Criminología un Ciencia?

\section{Conclusión}

Pese a los esfuerzos realizados en este trabajo por explicitar las dificultades a las cuales se enfrentan los investigadores al momento de determinar el estatus científico del saber criminológico y la propuesta de determinación sugerida, quedan aún demasiadas interrogantes por resolver, todas ellas capaces de dar un brusco giro a lo señalado anteriormente.

38 García-Pablos, Criminología: Una introducción a sus fundamentos teóricos, op. cit., pág. 36.

39 Lanier \& Stuart, Essential Criminology, op. cit., pág. 17; y Serrano, Introducción a la Criminología, op. cit., págs. 34-52.

40 Garrido, Stangeland y Redondo, Principios de Criminología, op. cit., págs. 55-59. 
Algunas de estas preguntas se vinculan principalmente con el aspecto multi o interdisciplinar de la criminología, y pueden formularse: si la Criminología se basa en diferentes disciplinas, ¿puede ella reivindicar tal condición? ${ }^{41}$, o la Criminología al ser parasitaria de otras disciplinas, ¿puede llamarse por sí misma?, o sería mejor considerarla como un campo de estudio más que una disciplina ${ }^{42}$; entonces, ¿es la Criminología simplemente la intromisión de diversas disciplinas en una materia particular? Estos cuestionamientos aumentan el desconcierto, pero aquello no debe nublar la perspectiva, y quizás sea más promisorio adoptar la posición de Radzinowics, para quien la Criminología no es una disciplina principal y autónoma, pues solo entra en la provincias de muchas otras ciencias versadas en la naturaleza humana y la sociedad; pero no por ello se debe olvidar que "... En alguna forma, muchas de las dudas que la Criminología ha fermentado para desafiar el statu quo son tan importantes como las explicaciones que ha exitosamente proporcionado..." 43 .

\section{Bibliografía}

Bunge, M. (1985). “¿Qué es la ciencia?” en M. Bunge, La ciencia su método y filosofía (págs. 8-36). Buenos Aires: Ediciones Siglo Veinte.

Cid Moliné, J. y Larrauri Pijoan, E. (2001). Teorías Criminológicas. Barcelona: Bosch.

Coleman, C. \& Norris, C. (2000). Introducing Criminology. Portland: Willan Publishing.

Corbetta, P. (2003). Metodología y técnicas de investigación social (M. Díaz Ugarte, y S. Díaz Ugarte, Trads.). Madrid: McGraw-Hill.

García-Pablos de Molina, A. (2005). Criminología: Una introducción a sus fundamentos teóricos ( $5^{\mathrm{a}}$ ed.). Valencia: Tirant lo Blanch.

Garrido, V., Stangeland, P. y Redondo, S. (2006). Principios de Criminología. Valencia: Tirant lo Blanch.

KAISER, G. (1988). Introducción a la Criminología (7a ed.). (N. J. Rodríguez y J. M. Rodríguez Devesa, Trads.). Madrid: Dykinson.

Lanier, M. M. \& Stuart, H. (2010). Essential Criminology (3a ed.). Philadelphia: Westview Press.

NAGEL, E. (1989). "La ciencia y el sentido común" en E. Nagel, La estructura de la ciencia (N. Miguez, Trad., págs. 15-26). Barcelona: Paidós.

41 Newburn, Criminology, op. cit., pág. 5.

42 Coleman \& Norris, Introducing Criminology, op. cit., pág 15.

43 Radzinowics, S. L. (1999). "A brief for Criminology" en S. L. Radzinowicz, Adventures in Criminology (págs. 440-469). London: Routledge. 
Newburn, T. (2007). Criminology. Portland: Willan Publishing.

Ove Hansson, S. (3 de septiembre de 2008). Sciencie and Pseudo-Science. Recuperado el 20 de marzo de 2010, de Stanford Encyclopedia of Philosophy: http://plato.stanford.edu/entries/pseudo-science/

Plantinga, A. (20 de febrero de 2007). Religion and Science. Recuperado el 1 de abril de 2010, de Stanford Encyclopedia of Philosophy: http://plato.stanford.edu/entries/religion-science/

Radzinowics, S. L. (1999). "A brief for Criminology" en S. L. Radzinowicz, Adventures in Criminology (págs. 440-469). London: Routledge.

RoldÁn Barbero, H. (2009). Introducción a la investigación criminológica. Granada: Editorial COMARES.

Serrano Maíllo, A. (2008). Introducción a la Criminología (5ª ed.). Madrid: Dykinson.

Tierney, J. (2006). Criminology: theory and context ( $2^{\text {a }}$ ed.). London: Pearson Education Limited. 